Research Article

\title{
Microbiological Isolates of Chronic Suppurative Otitis Media at the University Teaching Hospital and Beit Cure Hospital in Lusaka, Zambia
}

\author{
Harrison Phiri ${ }^{1, ~}$, Ayugi John ${ }^{1,2}$, Omutsani Mary ${ }^{1,3}$, Froeschl Uta ${ }^{4}$, Mwaba John $^{5}$ \\ ${ }^{1}$ Department of ENT, University of Nairobi, Nairobi, Kenya \\ ${ }^{2}$ Department of ENT, Head and Neck Surgery, University of Nairobi, Nairobi, Kenya \\ ${ }^{3}$ Department of ENT, Head and Neck Surgery, Kenyatta National Hospital, Nairobi, Kenya \\ ${ }^{4}$ Department of ENT, Head and Neck Surgery, Beit Cure Hospital, Lusaka, Zambia \\ ${ }^{5}$ Department of Microbiology, Bacteriology Unit, University Teaching Hospital, Lusaka, Zambia
}

Email address:

harridavis@yahoo.co.uk (H. Phiri)

${ }^{*}$ Corresponding author

\section{To cite this article:}

Harrison Phiri, Ayugi John, Omutsani Mary, Froeschl Uta, Mwaba John. Microbiological Isolates of Chronic Suppurative Otitis Media at the University Teaching Hospital and Beit Cure Hospital in Lusaka, Zambia. International Journal of Clinical and Experimental Medical Sciences. Vol. 2, No. 5, 2016, pp. 94-100. doi: 10.11648/j.ijcems.20160205.14

Received: June 7, 2016; Accepted: August 19, 2016; Published: September 6, 2016

\begin{abstract}
Background: Chronic Suppurative Otitis Media (CSOM) is a common cause of hearing loss and many complications such as meningitis. Many approaches to the treatment of CSOM have been unsatisfactory because CSOM microbiological isolates, as well as their sensitivity patterns, vary from place to place. This study sought to determine the pattern of microbiological isolates of CSOM and the demographic characteristics of patients with CSOM at the University Teaching Hospital, (UTH) and Beit Cure Hospital (BCH) in Lusaka, Zambia. Materials and Methods: The study was a hospital based Cross-sectional descriptive study conducted at the ENT outpatient clinics of UTH and BCH in Lusaka, Zambia.100 CSOM patients were included in the study. Quantitative data on the participants' demographic details and clinical features were obtained using structured questionnaires. The middle ear discharge was aseptically collected using a sterile cotton swab. In the laboratory, samples were inoculated on agar media to isolate microorganisms and antibiotic susceptibility testing was done using Kirby-Bauer method as per CLSI guidelines. Results: From the findings, out of the $100 \mathrm{CSOM}$ patients studied, 33(33\%) were children below 18yrs and $67(67 \%)$ were adults. 59(59\%) of the patients had unilateral CSOM while 41 had bilateral CSOM which gave a total of 141 ears that were analyzed.119(84.4\%) had pure cultures, 20(14.2\%) had mixed cultures and 2(1.4\%) had no growth. Of the 169 microbiological isolates, the most frequent isolates were Proteus mirabilis 49(29.0\%), Pseudomonas aeruginosa, 32(18.9\%), coagulase negative Staphylococcus 18(10.7\%) and klebsiella pneumoniae 17(10.1\%). High sensitivity rates were revealed to Gentamycin (64-100\%), meropenem (68-100\%), ceftazidime (85-100\%), ceftriaxone (64-80\%), and ciprofloxacin (66-88\%). High resistance rates were recorded to Amoxicillin-clavulanate (as high as 100\%), ampicillin (as high as $100 \%$ ), tetracycline (as high as $91.2 \%$ ) and cotrimoxazole (as high as 100\%) and penicillin (as high as 100\%). Conclusion: The study concluded that Proteus mirabilis was the most dominant microbiological isolate followed by Pseudomonas aeruginosa. The isolated microorganisms had high susceptibility rates to gentamycin, meropenem, ceftazidime, ceftriaxone and ciprofloxacin. There were high resistance rates to amoxicillin-clavulanate, ampicillin, tetracycline, cotrimoxazole and penicillin.
\end{abstract}

Keywords: Chronic Suppurative Otitis Media, Microbiological Isolates, Sensitivity, Resistance 


\section{Introduction}

Chronic suppurative otitis media (CSOM) is chronic inflammation of the middle ear cleft (Eustachian tube, middle ear, and mastoid cavity) which presents with recurrent ear discharge or otorrhoea through a tympanic perforation for two weeks or more. ${ }^{1} \mathrm{CSOM}$ commonly occurs during the first 6 years of a child's life, with a peak around 2 years. ${ }^{2}$ According to WHO, about 330million individuals, globally, have CSOM. In Africa, over 2.4 million people have CSOM, accounting for almost $4 \%$ of the global CSOM burden. ${ }^{1}$

The pathogenesis of CSOM is multifactorial with factors such as Eustachian tube (ET) dysfunction, genetic predisposition, and environmental factors playing a role. ${ }^{3}$ A dysfunctional and structurally immature ET is the most important factor in the pathogenesis of otitis media $(\mathrm{OM}){ }^{4}$ Infants and young children are especially at risk for reflux (containing bacteria and viruses) into the middle ear from nasopharynx via the ET because their ET is short, horizontal, and 'floppy'. ${ }^{3}$ The bacteria and viruses in the middle ear elicit an inflammatory response causing an acute infection. CSOM is initiated by an episode of acute infection of the middle ear that fails to resolve and result in a permanent TM perforation. 4

The common causative organisms of CSOM include aerobic bacteria such as Pseudomonas aeruginosa, Escherichia coli, Haemophilus influenza, Staphylococcus aureus and Klebsiella species. ${ }^{5,6}$ Anaerobic bacteria identified as CSOM causative organisms include Bacteroides and Fusobacteriumspecies. ${ }^{7}$ Aspergillus and Candidaspecies are common fungal isolates of CSOM. ${ }^{1,8}$ The sensitivity pattern of CSOM causative organisms varies generally from place to place due to differences in climatic conditions and manner of antibiotic use. ${ }^{9}$

Apart from being a cause of complications such as facial palsy, mastoiditis, brain abscess and labyrinthitis, CSOM is a major cause of acquired hearing impairment, especially in developing countries. ${ }^{1}$ Educational, vocational and social problems are some of the problems that stem from hearing impairment. Other problems include impaired speech and language development, poor academic performance and poor social interaction. ${ }^{1}$

\section{Materials and Methods}

This study was a hospital based cross-sectional descriptive study conducted in the ENT outpatient clinics at the university Teaching Hospital (UTH) and Beit Cure Hospital (BCH) which are situated in Lusaka, Zambia. Ethical approval was obtained from the KNH-UON Ethics Research Committee in Nairobi, Kenya; ERES (Excellence in Research and Science) Converge Ethical and Research Committee in Zambia; and from the aforementioned Hospitals. The sample size of 103 CSOM patients inclusive of $10 \%$ attrition was determined by the Yamane formula (1967:886)10 with a precision error of 5\%. The study group was as described under the section 2.1 below. The collection of materials for laboratory analysis and susceptibility tests have been described in section 2.2 and 2.3 respectively. Quality control was a continuous process throughout the study to maximize validity and reliability of the findings of the study. Measures that were put in place included the use of trained health professionals; aseptic techniques in collecting specimen; internal quality assessment of the procedures in the laboratory.

\subsection{Study Group}

The respondents were patients of all age groups who had actively draining CSOM (using WHO CSOM definition) and were attending the outpatient ENT clinics at UTH or BCH. They were patients who were not on any antibacterial and anti-fungal medication (at least in the last 2 weeks before participating in the study) and did not have any known immunosuppressive diseases. These were patients who consented or for whom a legal guardian had consented to participate in the study.

\subsection{Bacterial Isolation}

Using an aseptic technique (outlined below) pus discharge from the participants discharging ears was collected by the principal investigator within the ENT clinic on the first day of contact before any topical or systemic antibiotics or anti-fungal medication was started. Using sterile gloves (after washing hands with soap) under direct visualization with good lighting, and under microscopy for the majority of the patients, a sterile swab was passed through a sterile aural speculum placed in the EAC (to avoid contamination from the skin of the auditory canal) and then advanced to the middle ear or the inner two-thirds of the EAC to collect pus specimen. The sample obtained was put in a swab transport tube that contained a transport media and then labeled with a unique patient identifier and sent to the UTH microbiology laboratory. In the laboratory, by the laboratory technician, the specimens collected were inoculated on sheep Blood Agar, MacConkey's media, and chocolate agar media to culture aerobic bacteria. Anaerobic blood agar incubated in an anaerobic jar was used to culture anaerobic bacteria. Fungi were cultured on Sabouraud's dextrose agar. The culture plates were incubated at $37^{\circ} \mathrm{C}$ for 24-48 hours. Owing to the fact that anaerobes grow slowly compared to aerobes, anaerobic culture plates were incubated for up to 7 days to allow for anaerobic bacterial growth. Isolates from the culture plates were identified Using gram staining, colony morphology, catalase, coagulase, oxidase and biochemical strips. Lactophenol cotton blue was used for final identification of fungal growth.

\subsection{Antibiotic Susceptibility Testing}

Antimicrobial susceptibility tests were done on Mueller-Hinton agar using disk diffusion method as described by Kirby-Bauer. The antimicrobial agents tested were: tetracycline $(30 \mu \mathrm{g})$, chloramphenicol $(30 \mu \mathrm{g})$, gentamicin $(10 \mu \mathrm{g})$, ciprofloxacin $(5 \mu \mathrm{g})$, cotrimoxazole $(25 \mu \mathrm{g})$, ceftriaxone $(30 \mu \mathrm{g})$ and amoxicillin-clavulanate $(10 \mu \mathrm{g})$, meropenem, oxacillin, ceftazidime, cefoxitin, cefotaxime, 
ampicillin, and penicillin. Susceptibility data were interpreted according to Clinical and Laboratory Standards Institute (CLSI, 2015) by the microbiologist.

\subsection{Statistical Analysis}

Descriptive statistics were done using percentages of proportions, mean and standard deviation; Chi-square tests were done to establish relationships between variables using SPSS (Statistical Package for the Social Sciences) version 21.

\section{Findings}

\subsection{Demographic Characteristics and Clinical Findings of CSOM Patients}

A total of 100 CSOM patients were studied. The age range of the study patients was 6 months to 68 years. Of the patients studied, 33(33\%) were children under the age of 18, while $67(67 \%)$ were adults. The mean age was $24.5( \pm 18.0)$ years. The majority of the patients with CSOM in this study,81(81\%), resided in peri-urban areas which are associated with a low socioeconomic status. Other demographic characteristics of the respondents are as shown in Table 1.

Of the patientsstudied 59(59\%) had unilateral CSOM while 41 had bilateral CSOM which gave a total of 141 ears that were analyzed. Central perforation was the most common type of tympanic membrane perforation seen in $119(84.4 \%)$ ears, of which $43(30.5 \%)$ where subtotal. Attic perforations were seen in $2(1.4 \%)$ ears and marginal perforations in $9(6.4 \%)$ ears as shown in Table1.Granulation tissue was present in $20(14.2 \%)$ and cholesteatoma in $17(12.1 \%)$.

Table 1. Social-Demographic characteristics and clinical findings

\begin{tabular}{|c|c|c|c|}
\hline \multicolumn{4}{|l|}{ Social-Demographic characteristics } \\
\hline Characteristic & Category & Frequency & Percent \\
\hline \multirow{2}{*}{ Gender } & Male & 57 & 57.0 \\
\hline & Female & 43 & 43.0 \\
\hline \multirow{2}{*}{ Respondent } & Children & 33 & 33.0 \\
\hline & Adults & 67 & 67.0 \\
\hline \multirow{2}{*}{ Residence } & Urban & 19 & 19.0 \\
\hline & Peri-urban & 81 & 81.0 \\
\hline \multirow{5}{*}{ Level of education of adult patients } & Tertiary & 23 & 34.3 \\
\hline & Primary school & 15 & 22.4 \\
\hline & Junior secondary school & 15 & 22.4 \\
\hline & High school & 12 & 17.9 \\
\hline & Illiterate & 2 & 3.0 \\
\hline \multirow{3}{*}{ Occupation of patient } & Unemployed & 34 & 34.0 \\
\hline & Employed & 29 & 29.0 \\
\hline & Casual worker & 4 & 4.0 \\
\hline \multirow{3}{*}{ Occupation of parent } & Employed & 21 & 21.0 \\
\hline & Unemployed & 6 & 6.0 \\
\hline & Casual worker & 6 & 6.0 \\
\hline \multirow{3}{*}{ Size of household population } & $<6$ & 55 & 55.0 \\
\hline & $6-10$ & 40 & 40.0 \\
\hline & $>10$ & 5 & 5.0 \\
\hline \multirow{3}{*}{ Type of cooking fuel used } & Charcoal & 63 & 63.0 \\
\hline & Electricity & 35 & 35.0 \\
\hline & Firewood & 2 & 2.0 \\
\hline \multirow{2}{*}{ Household member smokes } & Does not smoke & 83 & 83.0 \\
\hline & Smokes & 17 & 17.0 \\
\hline \multirow{13}{*}{ Age groups } & $<=5$ & 19 & 19.0 \\
\hline & $6-10$ & 7 & 7.0 \\
\hline & $11-15$ & 7 & 7.0 \\
\hline & $16-20$ & 15 & 15.0 \\
\hline & $21-25$ & 7 & 7.0 \\
\hline & $26-30$ & 13 & 13.0 \\
\hline & $31-35$ & 6 & 6.0 \\
\hline & $36-40$ & 8 & 8.0 \\
\hline & $41-45$ & 5 & 5.0 \\
\hline & $46-50$ & 5 & 5.0 \\
\hline & $51-55$ & 2 & 2.0 \\
\hline & $61-65$ & 1 & 1.0 \\
\hline & $66-70$ & 5 & 5.0 \\
\hline \multicolumn{4}{|l|}{ Examination findings } \\
\hline \multirow{2}{*}{ Presence of Otorrhea } & Unilateral & 59 & 59.0 \\
\hline & Bilateral & 41 & 41.0 \\
\hline \multirow{4}{*}{ TM perforation } & Central & 119 & 84.4 \\
\hline & Subtotal & 43 & 30.5 \\
\hline & Total & 11 & 7.8 \\
\hline & Marginal & 9 & 6.4 \\
\hline
\end{tabular}




\begin{tabular}{llll}
\hline Social-Demographic characteristics & & & \\
\hline Characteristic & Category & Frequency & Percent \\
\hline & Attic & 2 & 1.4 \\
\hline
\end{tabular}

\subsection{Laboratory Findings}

Of the 141 specimens analyzed, 103(73.0\%) had gram negative rods, 22(15.6\%) had gram-positive cocci, 9(6.4\%) had gram-negative cocci, and $8(5.7 \%)$ had fungal elements. Pure cultures were $119(84.4 \%)$ and mixed cultures were $20(14.2 \%)$. 2(1.4\%) specimens had no growth. Of the 169 microbiological isolates obtained, the most dominant was Proteus mirabilis, 49(29.0\%). Other isolates are as shown in Table 2 below.

Table 2. Microbiological isolates.

\begin{tabular}{|c|c|c|c|}
\hline Microbiological Isolates & Oxygen requirement & & Percent \\
\hline Gram -ve Bacteria & Facultative anaerobes (n) & Aerobes (n) & \\
\hline Proteus mirabillis & 49 & & $29.0 \%$ \\
\hline Proteus vulgaris & 3 & & $1.8 \%$ \\
\hline Pseudomonas aeruginosa & & 32 & $18.9 \%$ \\
\hline Pseudomonas spp not aeruginosa & & 17 & $10.1 \%$ \\
\hline Klebsiela pneumoniae & 17 & & $10.1 \%$ \\
\hline Klebsiellaoxyntica & 2 & & $1.2 \%$ \\
\hline Corynebacterium & & 10 & $5.9 \%$ \\
\hline E.coli & 6 & & $3.6 \%$ \\
\hline Anterobacteragglomeraas & 2 & & $1.2 \%$ \\
\hline Actinomycetes & 1 & & $0.6 \%$ \\
\hline \multicolumn{4}{|l|}{ Gram +ve Bacteria } \\
\hline Staphylococus coagulase -ve spp & 18 & & $10.7 \%$ \\
\hline Staphylococcus aureus & 6 & & $3.6 \%$ \\
\hline Alpha hemolytic strep & 2 & & $1.2 \%$ \\
\hline Enteroccocusfeacalis & 1 & & $0.6 \%$ \\
\hline \multicolumn{4}{|l|}{ Fungi } \\
\hline Aspergillusniger & & 3 & $1.8 \%$ \\
\hline Total & 107 & 62 & $100 \%$ \\
\hline
\end{tabular}

\subsection{Susceptibility Tests}

Antibiotic susceptibility test was carried out for all the significant isolates which were Proteus mirabilis, Pseudomonas species, Klebsiella pneumoniae, and Coagulase Staphylococcus species. Proteus mirabilis showed high sensitivity rates with gentamycin $(91.3 \%)$, meropenem
(89.1\%), ceftazidime $(87.0 \%)$, ceftriaxone $(80.4 \%)$, cefotaxime (76.1\%), cefoxitin (65.2\%) and ciprofloxacin $(67.4 \%)$ as shown in Figure 1a below. Proteus mirabilis showed resistance rates of $77.8 \%$ to amoxicillin-clavulanate, $68.9 \%$ to ampicillin, $91.2 \%$ to tetracycline and $78.9 \%$ to cotrimoxazole (Figure 1a).

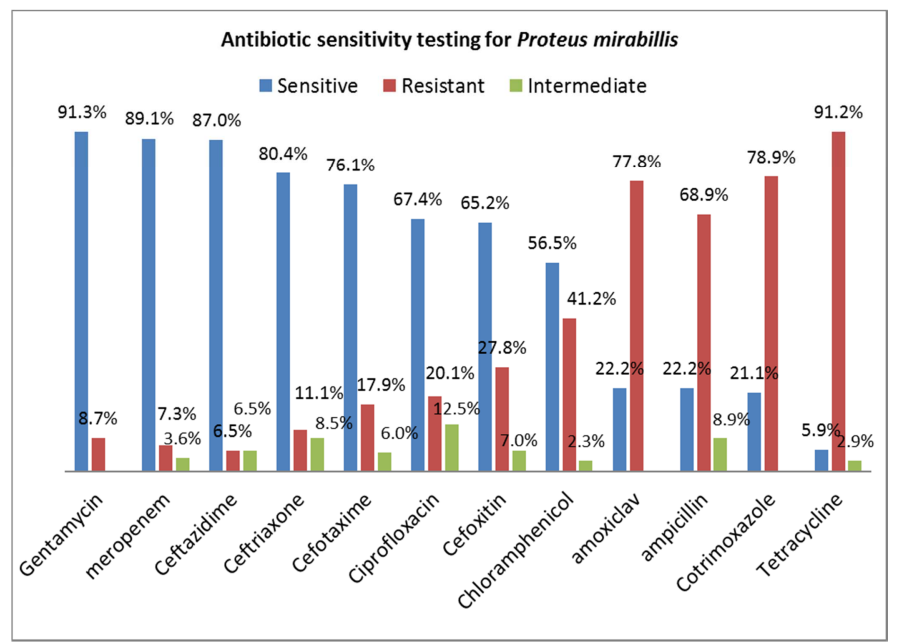

Figure 1a. Antibiotic Susceptibility testing for Proteus mirabilis.

Other gram-negative bacilli, Pseudomonas species, and Klebsiella pneumoniae, also showed high sensitivity rates for gentamycin (64-80\%, and 100\% respectively), meropenem
(66-93\%, and $100 \%$ respectively), ceftazidime ( $>90 \%$ and $80 \%$ respectively) and ciprofloxacin (66-88\%, and $84 \%$ respectively) as shown in Figure 1b, 1c and 1d below. 


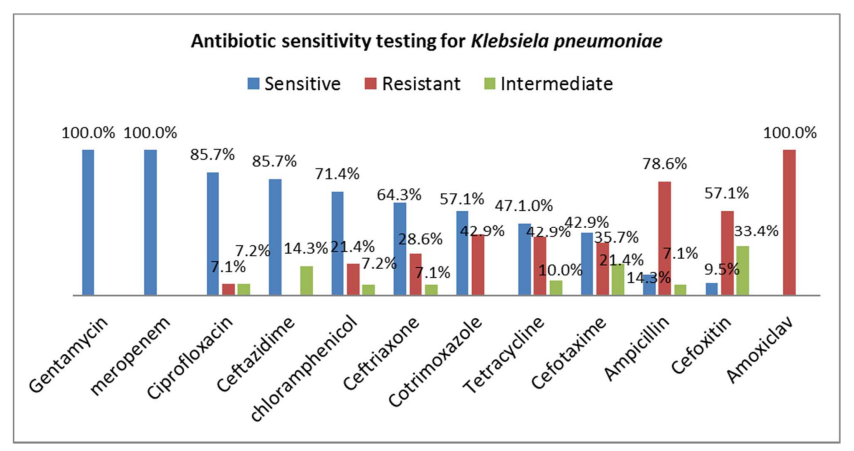

Figure 1b. Antibiotic susceptibility testing for Klebsiella pneumoniae.

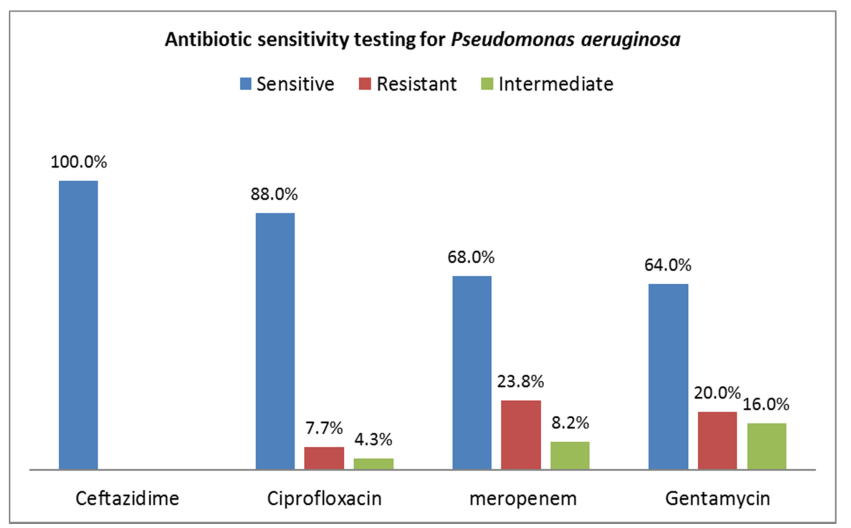

Figure 1c. Pseudomonas aeruginosa antibiotic susceptibility testing

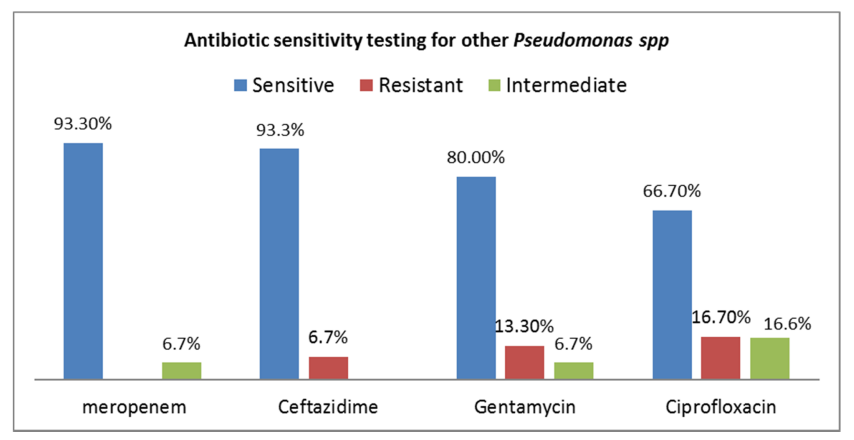

Figure 1d. Antibiotic susceptibility testing for other Pseudomonas species

High resistance rates were documented for gram-negative bacilli to amoxicillin-clavulanate, ampicillin, tetracycline, and cotrimoxazole (Figure 1b, 1c, 1d).

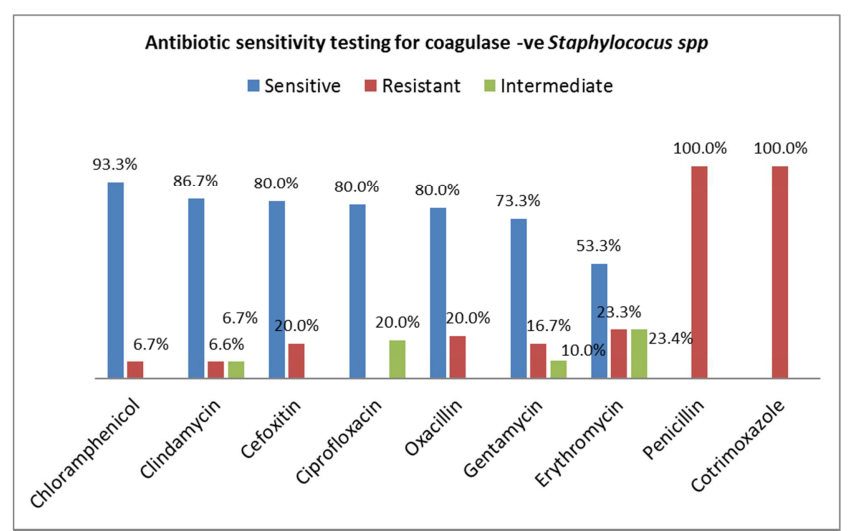

Figure 1e. Antibiotic susceptibility testing for coagulase negative staphylococcus species.
Gram positive cocci, coagulase negative staphylococcus species, showed high susceptible rates to gentamycin $(73.3 \%)$, oxacillin $(80 \%)$, cefoxitin $(80 \%)$, chloramphenicol $(93.3 \%)$, clindamycin $(86.7 \%)$ and ciprofloxacin $(80 \%)$ as shown in Figure 1e above. It showed the resistance of $100 \%$ to penicillin and co-trimoxazole.

\section{Discussion}

In this study of a 100 CSOM patients, the majority of the patients were adults that accounted for $67 \%$. Owing to the reason that $33 \%$ of the respondents were children and that 38 adult patients (56.7\% of the adults) reported onset of CSOM in childhood, it was inferred that CSOM is common in children. This is attributed to the short, wider and relatively horizontal Eustachian tube in this population. ${ }^{3}$

Children aged 0-5years accounted for $19 \%$ of the respondents (Table 1). This is similar to a study by Orji FT and Dike in 2015 where children below the age of 5 accounted for $23.8 \%$ of the patients. ${ }^{11}$ It was found that CSOM was slightly more common among male patients (57\%) similar to findings in a study done by Chirwa in 2014 in Malawi, where 64(61.5\%) were males. ${ }^{12}$ Other studies, however, found that CSOM was common among females than among males. ${ }^{13}$

The majority of the patients with CSOM in this study resided in peri-urban areas $81(81 \%)$ which are associated with a low socioeconomic status. This conforms to the notion that CSOM is a disease of those with a low socioeconomic status. $^{1,14}$

On examination, central perforation was the most common type of tympanic membrane perforation that was seen in 119 (84.4\%) ears. This implied that most of the patients had the safe type of CSOM, tubotympanic type, ${ }^{15}$ and may explain why there was no report of complications from the patients. A very small percentage of the ears had an attic perforation (1.4\%) and a marginal perforation $(6.4 \%)$ which explains the small number of cholesteatoma $(12 \%)$ that was found. Cholesteatoma was significantly associated with attic and marginal tympanic membrane perforation (Pearson coefficient $=1$ ).

The dominant microbiological isolate was Proteus mirabilis (29\%), a gram-negative facultative anaerobe (Table 2). This was followed by Pseudomonas aeruginosa (18.9\%) a gram negative aerobe. The finding of Proteus mirabilis as the most common isolate is similar to findings by Chirwa in 2014 in Malawi where Proteus mirabilis accounted for $28.6 \%,{ }^{12}$ Aduba et al in 2010inGarissa (Kenya) where Proteus mirabilis accounted for $32.7 \%$, ${ }^{16}$ and Muluye et alin 2013 in Ethiopia were Proteus mirabilis accounted for $27.5 \% .{ }^{17}$ These findings are different from those of other studies where they found that pseudomonas aeruginosa was the most common isolate. ${ }^{1,5,18}$ The difference in the pattern of microbiological isolates may be explained by differences in the geographical conditions and population dynamics. ${ }^{89}$ Proteus species are widely distributed in places with poor sanitary conditions, being found in feces, decomposing meat and sewage. ${ }^{19}$ This could account for its high frequency in this study where the majority of the patients $(81 \%)$ stayed in peri-urban areas which are 
associated with poor sanitary conditions.

In this study, there was no significant association between age distribution and pattern of microbiological isolates. It was however found in other studies that Proteus spp. were the commonest isolates in pediatrics compared to adults. ${ }^{20}$ There were no strict anaerobes that were isolated in this study. This finding differs from that in other studies were strict anaerobes were isolated. ${ }^{12,16}$ Some of the anaerobes isolated in other studies include Bacteroides species and Peptostreptococcus species.

Aspergillus niger, in only $3(1.8 \%)$ specimens, was the only fungal microorganism that was isolated in this study. The finding of Aspergillus niger as an etiological agent for CSOM is supported by other studies. ${ }^{12,21,22}$ In other studies, Candida species were also isolated. ${ }^{12,21}$

The sensitivity rates of Proteus mirabilis, gram-negative bacilli, for ciprofloxacin (a commonly used topical antibiotic), were relatively lower $(67.4 \%)$ than those found in the study by Bayeh et al in 2011where rates were as high as $93 \%{ }^{23}$ However, decreased sensitivity among gram-negative bacilli to Ciprofloxacin was also noted by Jeyakumari D. et al in $2015 .{ }^{24}$ Because Ciprofloxacin is the most commonly used otic antibiotic for CSOM, its lower sensitivity rates found in this study need to be further investigated.

As all the gram-negative bacilli, including proteus mirabilis, that were isolated in this study showed high susceptibility rates ( $>$ than $80 \%$ ) to Ceftazidime and meropenem, these drugs can be formulated as an empirical therapy for all gram-negative bacilli in cases of complicated CSOM where an intravenous drug would be required.

Comparable to our study, high resistance rates were reported for Proteus spp to tetracycline (100\%) and cotrimoxazole $(52 \%)$ by Wariso in Nigeria. ${ }^{25}$ Similarly, resistance rates of $89 \%$ for tetracycline and $64 \%$ for cotrimoxazole were reported by Bayer et al. ${ }^{23}$

Gram positive cocci, coagulase negative staphylococcus species, showed high susceptible rates to gentamycin $(73.3 \%)$, oxacillin $(80 \%)$, cefoxitin $(80 \%)$, chloramphenicol $(93.3 \%)$, clindamycin $(86.7 \%)$ and ciprofloxacin $(80 \%)$. These results are comparable with those in the study by Jeyakumari, D. et al where they found high sensitivity rates for staphylococcus species to clindamycin (93\%), Oxacillin (73\%), and ciprofloxacin $(73 \%) .{ }^{24}$ Due to the high susceptibility rates, these antibiotics can be designed as empirical therapy for Staphylococcus species. Coagulase negative staphylococcus spp showed $100 \%$ resistance rates to penicillin and cotrimoxazole. Jeyakumari D et al in 2015 also documented high resistance rates for Staphylococcus species to penicillin $(93 \%){ }^{24}$

\section{Conclusion}

CSOM is common in both children (33\%) and adults (67\%). It is more prevalent in the peri-urban areas $(81 \%)$ than in the urban areas (19\%). Proteus mirabilis (29\%), facultative gram-negative bacilli, was the most dominant microbiological isolate followed by Pseudomonas aeruginosa (18.9\%), a gram-negative aerobic bacilli. Other Pseudomonas species (not aeruginosa) (10.1\%) and Klebsiella pneumonia (10.1\%) were the other common gram-negative microbiological isolates. Coagulase negative staphylococcus species (10.7\%) were the most common gram-positive microbiological isolates The isolated microorganisms had high susceptibility rates to gentamycin (64-100\%), meropenem (68-100\%), ceftazidime $(85-100 \%)$, ceftriaxone (64-80\%), and ciprofloxacin (66-88\%). High resistance rates were recorded to Amoxicillin-clavulanate (as high as 100\%), ampicillin (as high as $100 \%$ ), tetracycline (as high as $91.2 \%$ ), cotrimoxazole (as high as $100 \%$ ) and penicillin (as high as $100 \%$ ). By virtue of having found a pattern of microbiological isolates in this study that is different from other studies, it can be inferred that culture and susceptibility testing for CSOM in a population/ geographical area is of paramount importance for appropriate antimicrobial therapy of CSOM.

\section{Acknowledgements}

University of Nairobi, in Kenya

Beit Cure Hospital in Lusaka Zambia

University Teaching Hospital in Lusaka, Zambia

Exactitude Research Consultants, Kenya

\section{Conflict of Interest}

None of the authors have any conflicts of interest associated with the work presented in this manuscript.

\section{References}

[1] Acuin J. Chronic Suppurative Otitis Media. Burden of Illness and Management Options. World Health Organization web site. http://www.who.int/pbd/chronicsuppurativeotitis_media.pdf. published in 2004.

[2] Daly K, Hunter L, Levine S, Lindgren B, Giebink G. Relationships between otitis media sequelae and age. Laryngoscope. 1998;108:1306-1310.

[3] Kenna M, Latz A. Otitis Media with Effusion. In: Bailey B, Johnson J, Newlands S, Ed. Head \& Neck Surgery Otolaryngology 4th Edition. Philadelphia, PA: Lippincott Williams \& Wilkins; 2006:1265-1271

[4] Probst R, Grevers G, Iro H. Basic Otorhinolaryngology: a step-by -step learning guide. Thieme. 2006:241-249.

[5] Afolabi O, Salaudeen A, Ologe F, Nwabuisi C, Nwawolo C. Pattern of bacterial isolates in the middle ear discharge of patients with chronic suppurative otitis media in a tertiary hospital in north central Nigeria. Afr Health Sci. 2012;12:362-367.

[6] Yeo S, Park D, Hong S, Cha C, Kim M. Bacteriology of chronic suppurative otitis media-a multicentre study. Acta Otolaryngol. 2007; 127:1062-1067.

[7] Brook I. The role of anaerobic bacteria in chronic suppurative otitis media in children: implications for medical therapy. Anaerobe. 2008;14:297-300. 
[8] Dawit F, Aberra G, Sileshi L, Abebe M. Drug susceptibility pattern of bacterial isolates from children with chronic suppurative otitis media. Ethiopian Journal of Health Development. 2001;15:89-96.

[9] Van-der-Veen E, Schilder A, Van-Heerbeek N, et al. Predictors of Chronic Suppurative Otitis Media in Children. Arch Otolaryngol Head Neck Surg. 2006;132:1115-1118.

[10] Yamane T. Statistics, An Introductory Analysis, $2^{\text {nd }}$ Edition, New York: Harper and Row. 1967.

[11] Orji FT, Dike BO. Observations on the current bacteriological profile of chronicsuppurative otitis media in South Eastern Nigeria. Ann Med Health Sci Res. 2015;5:124-128.

[12] Chirwa M. Microbiology of Chronic Otitis Media at Queen Elizabeth Central Hospital, Blantyre Malawi [dissertation]. Nairobi, Kenya: University Of Nairobi; 2014.

[13] Prakash R, Juyal D, Negi V, et al. Microbiology of chronic suppurative otitis Media in a tertiary care setup of Uttarakhand state, India. North American Journal of Medical Sciences. 2013;5:282-287.

[14] Kenna M. Etiology and pathogenesis of chronic suppurative otitis media. Arch Otolaryngol Head and Neck Surg. 1988;97:16-17.

[15] Balasubramanian T. Chronic suppurative otitis media. otolaryngology online. http//www.drtbalu.com/otology.htm. published in 2006.

[16] Aduda D, Macharia I, Mugwe P, et al. Bacteriology of chronic suppurative otitis media (CSOM) in children in Garissa district, Kenya: a point prevalence study. Int J PediatrOtorhinolaryngol. 2013;77:1107-1111.

[17] Muluye D, Wondimeneh Y, Ferede G, Moges F, Nega T. Bacterial isolates and drug susceptibility patterns of ear discharge from patients with ear infection at Gondar University Hospital, Northwest Ethiopia. BMC Ear Nose Throat Disord. 2013;13:10.
[18] Hatcher J, Smith A, Mackenzie I, et al. A prevalence study of ear problems in school children in Kiambu district, Kenya. Int J Pediatr Otorhinolaryngol. 1995;33:19.

[19] Hawkey p. Proteus, Providencia and Morganella spp. In: Gillespie S, Hawkey P, ed. Principles and Practice of Clinical Bacteriology. $2^{\text {nd }}$ ed. West Sussex: John Wiley and sons; 2006:392-396

[20] Yismaw G, Abay S, Asrat D, Yifru S, Kassu A. Bacteriological profile and resistance patterns of clinical isolates from pediatric patients, Gondar University teaching hospital. Ethiop Med J. 2010;48(4):293-300.

[21] Mwaniki R. Evaluation of Bacterial Flora and Antimicrobial Susceptibility of Chronic Otitis Media at Kenyatta National Hospital, Kenya [dissertation]. Nairobi, Kenya: University of Nairobi; 2009.

[22] Ibekwe A, Al-Shareef Z, Benayam A. Anaerobes and fungi in chronic suppurative otitis media. Ann OtolRhinolLaryngol. 1997;106:649-652.

[23] Bayeh A, Mulugeta K. Bacteriology and Antimicrobial Susceptibility of Otitis Media at Dessie Regional Health Research Laboratory, Ethiopia. Ethiopian Journal of Health Development. 2011;25:161.

[24] Jeyakumari D, Saranya R, Nagajothi J, Selvalingam E. Clinical and bacteriological profile of chronic suppurative otitis media in a rural area of puducherry. International Journal of Development Research. 2015;5:5518-5522.

[25] Wariso B, Ibe S. Bacteriology of chronic discharging ears in Port Harcourt, Nigeria. WAJM. 2006;25. 